\title{
Shared Bacterial and Viral Respiratory Agents in Bighorn Sheep (Ovis canadensis), Domestic Sheep (Ovis aries), and Goats (Capra hircus) in Montana
}

\author{
David S. Miller, ${ }^{1}$ Glen C. Weiser, ${ }^{2}$ Keith Aune, ${ }^{3,4}$ Brent Roeder, ${ }^{5}$ Mark Atkinson, ${ }^{3,6}$ \\ Neil Anderson, ${ }^{3}$ Thomas J. Roffe, ${ }^{7}$ Kim A. Keating, ${ }^{8}$ Phillip L. Chapman, ${ }^{9}$ Cleon Kimberling, ${ }^{1}$ \\ Jack Rhyan, ${ }^{10}$ and P. Ryan Clarke ${ }^{11}$ \\ ${ }^{1}$ Department of Clinical Sciences, College of Veterinary Medicine and Biomedical Sciences, Colorado State University, \\ Fort Collins, CO 80523, USA \\ ${ }^{2}$ University of Idaho, College of Agricultural and Life Sciences, Caine Veterinary Teaching Center, 1020 East Homedale Road, \\ Caldwell, ID 83607, USA \\ ${ }^{3}$ Montana Fish, Wildlife \& Parks, 1400 South 19th Avenue, Bozeman, MT 59715, USA \\ ${ }^{4}$ Wildife Conservation Society, 2023 Stadium Drive, Suite 1A, Bozeman, MT 59715, USA \\ ${ }^{5}$ Department of Animal \& Range Sciences, Montana State University, P.O. Box 172900, Bozeman, MT 59717, USA \\ ${ }^{6}$ Wildlife Conservation Society, 2300 Southern Boulevard, Bronx, NY 10460, USA \\ 7 U.S. Fish and Wildlife Service, 1400 S 19 th Avenue, Bozeman, MT 59718, USA \\ ${ }^{8}$ U.S. Geological Survey, Northern Rocky Mountain Science Center, 2327 University Way, Suite 2, Bozeman, MT 59715, USA \\ ${ }^{9}$ Department of Statistics, Colorado State University, 224 Statistics Building, Fort Collins, CO 80523, USA \\ ${ }^{10}$ National Wildlife Research Center, 4101 La Porte Avenue, Fort Collins, CO 80521, USA \\ ${ }^{11}$ Animal and Plant Health Inspection Service, U.S. Department of Agriculture, Western Region, Belgrade, MT 59714, USA
}

Correspondence should be addressed to David S. Miller, dsmdaczm@gmail.com

Received 18 July 2011; Accepted 24 August 2011

Academic Editor: Michael Stoskopf

Copyright () 2011 David S. Miller et al. This is an open access article distributed under the Creative Commons Attribution License, which permits unrestricted use, distribution, and reproduction in any medium, provided the original work is properly cited.

\begin{abstract}
Transmission of infectious agents from livestock reservoirs has been hypothesized to cause respiratory disease outbreaks in bighorn sheep (Ovis canadensis), and land management policies intended to limit this transmission have proven controversial. This crosssectional study compares the infectious agents present in multiple populations of bighorn sheep near to and distant from their interface with domestic sheep (O. aries) and domestic goat (Capra hircus) and provides critical baseline information needed for interpretations of cross-species transmission risks. Bighorn sheep and livestock shared exposure to Pasteurellaceae, viral, and endoparasite agents. In contrast, although the impact is uncertain, Mycoplasma sp. was isolated from livestock but not bighorn sheep. These results may be the result of historic cross-species transmission of agents that has resulted in a mosaic of endemic and exotic agents. Future work using longitudinal and multiple population comparisons is needed to rigorously establish the risk of outbreaks from cross-species transmission of infectious agents.
\end{abstract}

\section{Introduction}

Bighorn sheep (Ovis canadensis) experienced substantial decreases in population numbers and range in the 19th and the early 20th centuries, and subsequent recovery efforts have often been limited by large-scale die-offs [1-3]. These initial population declines were associated with settlement of western North America and were attributed to unregulated hunting, competition for forage with domestic sheep (O. aries) and other livestock, and disruption of historic bighorn sheep migration patterns due to development. Clinical disease was apparently unimportant or was underreported in these early declines, though die-offs of bighorn sheep associated with sheep scab (Psoroptes sp.) were reported following settlement [4, $5]$. 
Bighorn sheep die-offs associated with pneumonia were reported in the 1920s and 1930s [6-10]. These early reports and subsequent work largely focused on lungworm (Protostronglus $\mathrm{sp}$.) as the primary infectious agent, although the involvement of Pasteurella sp., Corynebacterium pyogenes (currently Arcanobacterium pyogenes), and other host and environmental determinants were also noted as potential causes of respiratory disease. Subsequently, inconsistent association of lungworm with respiratory disease in bighorn sheep, as well as further evidence for Pasteurella sp. as the cause of pneumonia, led to a focus on pasteurellosis as a cause of respiratory disease outbreaks [11-14]. This research included evidence that Pasteurella sp. strains from clinically normal domestic sheep were pathogenic to bighorn sheep, and a molecular basis for this observation was established [15-17]. Much of this research was conducted under captive conditions or in vitro, due to the challenges of identifying morbid or recently dead animals that are appropriate for sampling, variation in methods for investigating outbreaks, and other challenges for conducting field investigations on bighorn sheep diseases. Recent evidence confirms that transmission of Mannheimia haemolytica (formerly Pasteurella haemolytica) from domestic sheep to bighorn sheep can occur under experimental conditions when comingling [18]. Alternative hypotheses that are not mutually exclusive with pasteurellosis include other infectious agents, external stressors, and nutritional deficiencies that can lead to compromised bighorn sheep immunity to infectious disease [1921]. An understanding of the etiopathogenesis of bighorn sheep respiratory disease outbreaks is further complicated by inconsistent isolation of agents such as Mycoplasma ovipneumoniae and viruses from bighorns during such outbreaks $[22,23]$. In addition, pneumonia in lambs has been described as a distinct phenomenon from that of adults [9, 24]. Understanding the complex etiopathogenesis of bighorn sheep bronchopneumonia in adult and juvenile bighorn sheep may provide managers with options to mitigate, halt, or prevent some bighorn sheep die-offs.

The first step to better understand the complex relationships in the etiopathogenesis of bronchopneumonia is to identify the normal suite of viruses, bacteria, and parasites in healthy bighorn sheep and livestock populations. This report provides baseline data for Pasteurellaceae biovariants, Mycoplasma sp., selected viral respiratory agents, and endoparasites from multiple populations of apparently healthy bighorn and domestic sheep.

\section{Materials and Methods}

2.1. Populations Studied. Bighorn sheep populations were sampled opportunistically when management or research objectives permitted. Domestic sheep populations were sampled based on producer permission and whether located distant from or near known bighorn sheep but were not meant to be all inclusive for all populations at a given location. Four different types of populations were sampled using consistent methods: isolated and interface bighorn sheep populations and isolated and interface domestic sheep populations (Table 1). Isolated and interface populations were classified based on the location where the population was sampled, without inference as to historic movement of individuals or populations. Isolated domestic sheep populations consisted of populations that had one of the following characteristics: (a) bighorn sheep were not known to be within $14.5 \mathrm{~km}$, or (b) bighorn sheep were prevented from commingling with domestics by physical barriers (housing development), (c) bighorn sheep were temporally separated from domestics by season of occupation. Bighorn sheep were reported by producers to be in pastures or within visual contact of pastures for each interface livestock population studied. For each interface bighorn sheep population, two nearby domestic sheep populations were identified for the purpose of evaluating shared virus, bacteria, and parasitic agents. One goat population that was comanaged with an interface domestic sheep population was also included in the study, due to the potential for transmission of agents between these species. The number of animals sampled in each population varied due to availability, and cost constraints limited testing of all animals for all agents. The threshold of $14.5 \mathrm{~km}$ distance was initially used for classifying "isolated" populations based on management guidelines for bighorn and domestic sheep, although subsequently superseded by visual observations of pasture contact [25]. Consequently, interface populations had pasture or closer contact, whereas isolated populations were those where pasture or closer contact was considered unlikely.

Isolated bighorn sheep populations included those located in or near Thompson Falls, Perma/Plains, Sun River, Charles M. Russell National Wildlife Refuge, National Bison Range, Glacier National Park, and Harper's Ferry (Montana Fish Wildlife and Parks (MFWP) population HD622), Montana [26]. Interface bighorn sheep populations included those near Winifred, Anaconda, and Helena, Montana. Domestic sheep population identification was coded due to participant confidentiality concerns. Locations for populations were recorded in World Geodetic System (WGS) 84 GPS format.

Bighorn sheep populations were characterized from winter aerial surveys conducted by MFWP in 2003. Domestic livestock populations were characterized from questionnaires verbally administered to the producers after sampling their flock.

2.2. Animal Handling and Sampling. Animal handling research protocols were approved by Institutional Animal Care and Use Committees at Colorado State University (protocol number ACUC 05-05-283A-01) and the US Geologic Survey's Northern Rocky Mountain Science Center (unnumbered protocol for work conducted in Glacier National Park). Bighorn sheep were captured in 2002-2006 during the months of September-June, as a part of routine research and management activities, and were conducted using a combination of physical (helicopter net gun) and chemical restraint techniques [27]. Domestic livestock were manually restrained during the spring or fall-2005-2006. Physical examination and biomedical sample collection procedures 
TABLE 1: Characteristics of bighorn sheep and domestic sheep populations studied, based on proximity to the bighorn/domestic sheep interface.

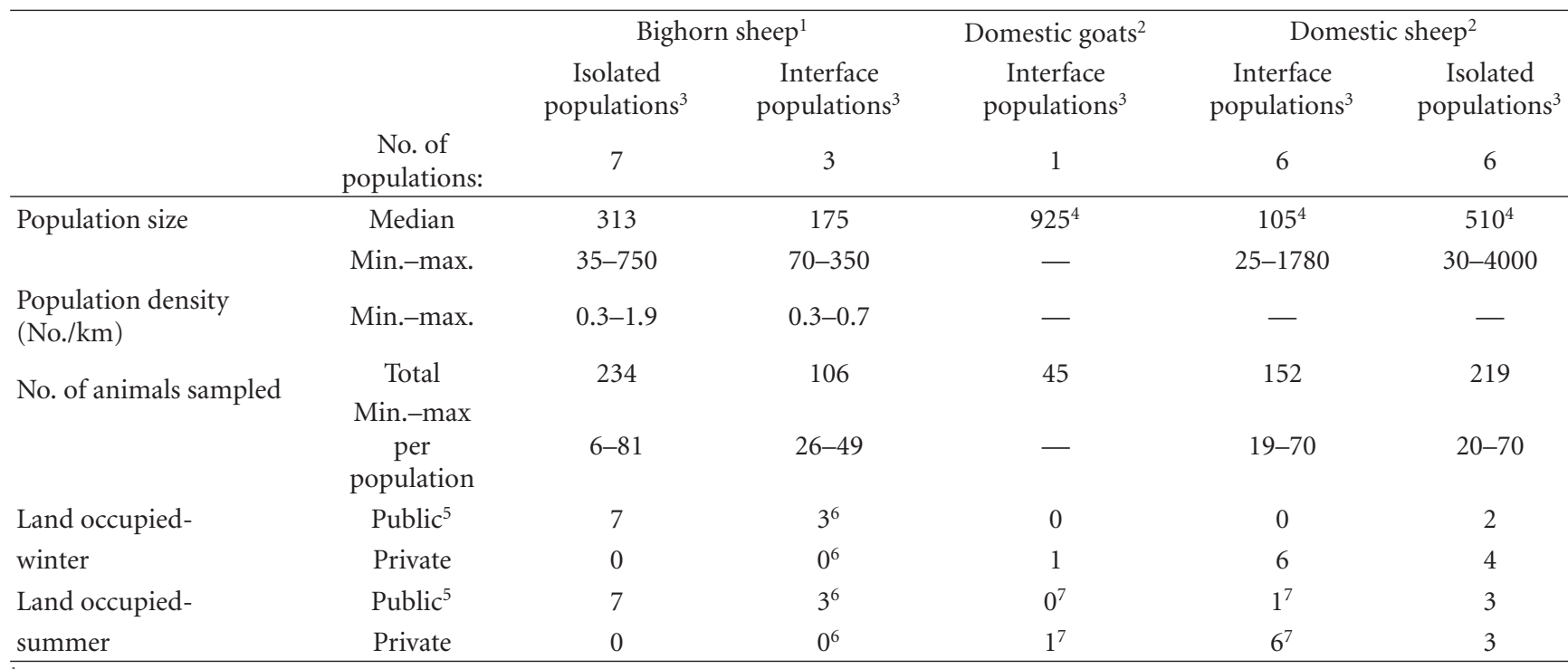

${ }^{1}$ Data based on 2003 annual aerial census by Montana Fish, Wildlife and Parks.

${ }^{2}$ Data from questionnaire.

${ }^{3}$ Based on $14.5 \mathrm{~km}$ barrier recommended for land management [25]; interface $\leq 14.5 \mathrm{~km}$, relative to sympatric species, and isolated $>14.5 \mathrm{~km}$, relative to sympatric species (or surrounded by development that prevents interactions with sympatric species).

${ }^{4}$ Number of females in population.

${ }^{5}$ Public land: federal and state lands.

${ }^{6}$ One population $50 \%$ federal and $50 \%$ private land.

${ }^{7}$ One population $10 \%$ on public land.

were comparable for all animals and were conducted as quickly as possible to minimize overheating and capture stress. Snow, water, or ethanol was applied to bighorn sheep to correct hyperthermia, as needed. Evidence of respiratory disease was noted, including nasal discharge or coughing. Uniquely identified individuals were resampled in three isolated domestic sheep populations and one goat population on two occasions, $6 \mathrm{mo}$ apart, to determine temporal variability. All samples were uniquely identified by population, date, and individual.

Pasteurellaceae biovariants and subspecies (no. of isolates) cultured from individual isolated and interface bighorn sheep ( $n=10$ populations), domestic sheep $(n=12$ populations), and goats ( $n=1$ population).

Sampling of the oropharynx of all animals for bacteria was conducted by opening the oral cavity with a mouth gag cleaned in soapy water and disinfected in $70 \%$ ethanol or by hands covered with fresh, disposable gloves per standardized protocols developed by the Western Wildlife Health Committee, Association of Western Fish and Wildlife Agencies [28]. A Dacron swab was used to sample the surface of the palatine tonsils and surrounding oropharyngeal region using methods developed for bighorn sheep [28]. The swab was immediately placed in sterile media tubes containing modified Cary Blair media (Port-a-cul, Becton-Dickinson, Franklin Lakes, NJ, USA) and placed on cold packs. The swab was shipped chilled without freezing to a reference laboratory (Caine Veterinary Teaching Center, University of Idaho, Caldwell, ID, USA) (CVTC) for Pasteurellaceae sp. and Mycoplasma sp. culture within $72 \mathrm{hr}$ of collection. Swabs that contacted the tongue, teeth, or other potential sites of contamination were discarded, and the process was repeated until a sample representative of the oropharyngeal flora was collected.

Blood was collected via jugular venipuncture into sterile serum collection tubes (Vacutainer, Becton-Dickinson, Franklin Lakes, NJ, USA). Blood samples were kept cool in the field by using cold packs. Samples were subsequently centrifuged the day of collection, and serum was removed. Serum was hand carried on cool packs or shipped frozen serum to the Montana Veterinary Diagnostic Laboratory (Bozeman, MT, USA) (MVDL) for viral serology.

Feces were collected from the rectum or upon defecation during processing. Samples were kept chilled and submitted to the veterinary diagnostic laboratory for fecal floatation and Baermann analyses $[29,30]$.

Following Montana Fish Wildlife and Parks policy, a male bighorn sheep found to be commingling with domestic sheep and goats in animal shelters for more than six months was euthanized using approved procedures [31]. An oral swab and lung tissue were collected from this individual for Pasteurellaceae and Mycoplasma culture. Oral swabs were collected from commingling domestic sheep $(n=29)$ and domestic goats $(n=34)$.

2.3. Bacterial Culture and Classification Procedures. Consistent culture methods were used throughout the study over time and between samples sources. At CVTC, the 
oropharyngeal swab from each animal was inoculated onto nonselective Columbia blood agar (CBA) (Becton Dickinson \& Co., Sparks, MD, USA), containing 5\% sheep blood, and CBA with selective antibiotics, containing 5\% bovine blood [32], and incubated for $24 \mathrm{hr}$ at $37^{\circ} \mathrm{C}$ in a $10 \%$ $\mathrm{CO}_{2}$ atmosphere. Following incubation, representatives of each colony type were propagated on fresh CBA for species and Pasteurellaceae biovariant classification using previously described methods that are useful for wildlife studies [33, 34]. For the purposes of this report, each distinct bacteria that was identified among cultures from an oropharyngeal swab is called an isolate, even if multiple colonies of that bacteria were cultured from a single swab.

At CVTC, the swab was subsequently placed in Mycoplasma enrichment broth [35] and incubated at $37^{\circ} \mathrm{C}$ for $72 \mathrm{hr}$ using methods that have previously identified M. ovipneumoniae in bighorn sheep [36]. Broth was subsequently streaked on Mycoplasma plates and incubated at $37^{\circ} \mathrm{C}$ with $5-10 \%$ $\mathrm{CO}_{2}$ for 5-7 days. Finally, Mycoplasma colonies were selected and plated on fresh medium. Not every sample was processed for Mycoplasma, but every population had a minimum of five animals cultured for Mycoplasma.

2.4. Serology Procedures. Serology was conducted at MVDL for antibodies to viruses with the potential to cause or predispose animals to respiratory infection. The serum neutralization (SN) test was used for infectious bovine rhinotracheitis (IBR) virus, bovine viral diarrhea virus (BVDV-1 and BVDV2), and bovine respiratory syncytial virus (BRSV) [37-39] and hemagglutination inhibition for parainfluenza-3 (PI-3) $[38,40]$. These methods classified serology titer results $\geq 1: 8$ as positive for antibodies to IBR, BVDV-1, BVDV-2, BRSV, and PI-3. Seroconversion was defined as a $\geq$ fourfold increase in titer for any of the four viruses.

2.5. Fecal Parasitology. Veterinary Parasitology Services used conventional fecal floatation to recover oocysts and eggs of gastrointestinal nematodes, cestodes, and protozoa, whereas Baermann assay methods were used to separate first-stage larvae of lungworms prior to identification [30]. Parasites were qualitatively reported as present or absent, without quantification. Eggs of Trichostongylina were not differentiated by genus. Larvae of Protostrongylidae and Dictyocaulidae strongylid larvae were identified to genus. Cost constraints prevented conducting assays on all animals.

2.6. Statistical Analyses. Individuals were classified as positive or negative for specific agents based on the results of bacterial culture, viral serology, and fecal parasitology. For data collected multiple times from an individual, data from the first sampling event was used, except for temporal analyses.

Statistical analysis was conducted using SAS 9.2 (SAS Institute, Inc., Cary, NC, USA). Chi-square analyses were conducted using the FREQ procedure with the threshold for significance set at $P \leq 0.05$. Odds ratios (ORs) were calculated for 2 by 2 tables. Chi-square tests assume independence of counts. Separate Chi-square analyses were conducted for each host species' Pasteurellaceae isolates to evaluate associations between the host population's interface status (i.e., whether the host population is at an interface or is isolated) and whether isolates were beta-hemolytic (which often is interpreted as presumptive evidence of pathogenicity).

\section{Results}

3.1. Populations Studied. Population sizes for sampled bighorn and domestic sheep varied (Table 1). Bighorn sheep primarily inhabited public land, whereas domestic sheep primarily resided on private land for operations managed for wool, meat, or mixed objectives. Bighorn sheep were $1-14 \mathrm{y}$, and domestic sheep were 1-10y. Domestic sheep with evidence of mild respiratory disease were found in four interface populations $(n=10)$ and one isolated population $(n=1)$. No domestic goats or bighorn sheep had evidence of respiratory disease.

3.2. Bacteriology: Pasteurellaceae. Two hundred sixty-five bighorn sheep, 203 domestic goat, and 790 domestic sheep Pasteurellaceae isolates were identified (Table 2). These isolates comprised 166 unique Pasteurellaceae species or biovariants that were often identified in multiple host species. Bighorn sheep, domestic goats, and domestic sheep had 60, 37, and 135 different Pasteurellaceae species or biovariants isolated, respectively. Thirty six of the bighorn sheep Pasteurellaceae species or biovariants were also found in domestic livestock, and this overlap represented 72\% $(n=$ $190)$ of the bighorn sheep, $82 \%(n=167)$ of domestic goat, and 58\% $(n=462)$ of domestic sheep Pasteurellaceae isolates. Bighorn sheep isolates were primarily (73\%) P. (B.) trehalosi $(n=193)$, whereas most $(60 \%)$ of domestic sheep isolates were M. haemolytica $(n=473)$. Half $(50 \%)$ of domestic goat isolates were $P$. (B.) trehalosi $(n=102)$, and $44 \%$ were M. haemolytica $(n=89)$. There were also 375 bighorn sheep, 96 domestic goat, and 448 domestic sheep isolates that were not characterized as Pasteurellaceae, including Arcanobacterium pyogenes, Bacillus sp., Enterobacter sp., Enterococcus sp., Moraxella sp., Neisseria sp., Proteus sp., Pseudomonas sp., Staphylococcus sp., Streptococcus sp., and coliforms.

Twenty-two biovariants were found in interface bighorn sheep populations but not in isolated populations. For comparison, 24 biovariants were found in isolated bighorn sheep populations but not in interface populations. Among domestic sheep, 38 biovariants were found only in interface populations, and 52 biovariants were found only in isolated populations. There was not a significant association between whether a Pasteurellaceae isolate was beta-hemolytic and whether the isolate was collected at the interface for domestic $(P=0.89$; OR $0.98,95 \%$ CI $0.71-1.34)$ or bighorn sheep populations ( $P=0.41$; OR $0.76,95 \%$ CI $0.40-1.45)$.

Individual domestic sheep $(n=85)$ and goats $(n=34)$ from three sheep and one goat operation were resampled 6 mo apart to assess temporal variation in Pasteurellaceae biovariants. None of the domestic sheep and goats sampled 
TABLE 2: Pasteurellaceae biovariants and subspecies (no. of isolates) cultured from individual isolated and interface bighorn sheep $(n=10$ populations), domestic sheep ( $n=12$ populations), and goats ( $n=1$ population).

\begin{tabular}{|c|c|c|c|c|c|}
\hline \multirow{2}{*}{$\begin{array}{l}\text { Host species: } \\
\text { Interface }^{1}\end{array}$} & \multicolumn{2}{|c|}{ Bighorn sheep } & \multirow{2}{*}{$\begin{array}{l}\text { Goat } \\
\text { Yes }\end{array}$} & \multicolumn{2}{|c|}{ Domestic sheep } \\
\hline & No & Yes & & Yes & No \\
\hline No. of populations & 7 & 3 & 1 & 6 & 6 \\
\hline No. of animals: & 234 & 105 & 45 & 152 & 219 \\
\hline No. of Pasteurellaceae isolates & 144 & 121 & 203 & 389 & 401 \\
\hline \multicolumn{6}{|l|}{ Species } \\
\hline Actinobacillus & 1 & & 6 & 35 & 16 \\
\hline \multirow[t]{11}{*}{ Mannheimia haemolytica } & $1(1), 1^{\alpha} \quad(2)$ & $1(1), 1^{\alpha}(1), 1^{G}(2)$ & $\begin{array}{c}1(18), 1^{\alpha}(2), \\
1^{\alpha \mathrm{B}}(1), 1^{\alpha \mathrm{G}}(1), \\
1^{\mathrm{B}}(1), 1^{\mathrm{E}}(10), \\
1^{\mathrm{EG}}(1), 1^{\mathrm{G}}(5)\end{array}$ & $\begin{array}{c}1(21), 1^{\alpha}(4), \\
1^{\alpha \mathrm{B}}(1), 1^{\alpha \mathrm{BG}}(2), \\
1^{\alpha \mathrm{GG}}(1), 1^{\mathrm{EG}}(1), \\
1^{\mathrm{G}}(11)\end{array}$ & $\begin{array}{c}1(28), 1^{\alpha}(4), \\
1^{\alpha \mathrm{B}}(1), 1^{\alpha \mathrm{E}}(1), \\
1^{\alpha \mathrm{G}}(1), 1^{\mathrm{E}}(1), \\
1^{\mathrm{EG}}(1), 1^{\mathrm{G}}(8)\end{array}$ \\
\hline & $\begin{array}{c}3^{\alpha \mathrm{BCE}}(1), \\
3^{\alpha \mathrm{BG}}(1), \mathrm{B}(2)\end{array}$ & $\begin{array}{c}3^{\alpha \mathrm{CD}}(1) \\
3^{\mathrm{CDE}}(2), 3^{\mathrm{G}}(1)\end{array}$ & $\begin{array}{c}3(3), 3^{\beta}(1) \\
3^{\beta \mathrm{BDE}}(1), 3^{\mathrm{C}}(1)\end{array}$ & $\begin{array}{c}3(17), 3^{\alpha}(1), \\
3^{\alpha \mathrm{G}}(1), 3^{\beta}(2), \\
3^{\beta \mathrm{CDE}}(1), 3^{\mathrm{C}}(1), \\
3^{\mathrm{CD}}(1), 3^{\mathrm{CE}}(1), \\
3^{\mathrm{DE}}(1), 3^{\mathrm{E}}(5)\end{array}$ & $\begin{array}{c}3(18), 3^{\alpha}(1), \\
3^{\alpha \mathrm{BDE}}(1), 3^{\alpha \mathrm{BDE}}(1), \\
3^{\alpha \mathrm{BDE}}(1), 3^{\beta \mathrm{CD}}(1), \\
3^{\beta \mathrm{D}}(2), 3^{\mathrm{D}}(1) \\
3^{\mathrm{E}}(1)\end{array}$ \\
\hline & & & & $\begin{array}{c}5(9), 5^{\alpha}(2), \\
5^{\alpha \mathrm{B}}(2), 5^{\alpha \beta \mathrm{G}}(1) \\
5^{\mathrm{C}}(1)\end{array}$ & $\begin{array}{c}5(12), 5^{\alpha \mathrm{B}}(2), \\
5^{\alpha \beta \mathrm{BD}}(1), 5^{\mathrm{B}}(4), \\
5^{\beta \mathrm{BD}}(2), 5^{\beta \mathrm{B}}(1), \\
5^{\mathrm{D}}(1)\end{array}$ \\
\hline & $6^{\alpha \mathrm{R}}(1)$ & $6^{\alpha \mathrm{R}}(1)$ & & & $6^{\alpha \mathrm{R}}(2)$ \\
\hline & & & $7(3), 7^{\mathrm{X}}(2)$ & $\begin{array}{c}7(3), 7^{\mathrm{B}}(1), \\
7^{\mathrm{BX}}(1), 7^{\mathrm{X}}(5)\end{array}$ & $\begin{array}{c}7(4), 7^{\mathrm{B}}(6), \\
7^{\mathrm{BGX}} 7(1), 7^{\mathrm{BX}}(3), \\
7^{\mathrm{X}}(3)\end{array}$ \\
\hline & $8(1)$ & $8(1), 8^{\mathrm{B}}(1)$ & $8(6)$ & $8(7), 8^{B}(6)$ & $\begin{array}{c}8(19), 8^{\mathrm{B}}(3), \\
8^{\mathrm{G}}(2)\end{array}$ \\
\hline & $9^{\alpha \beta B}(1)$ & $\begin{array}{c}9^{\alpha}(1), 9^{\alpha \beta \mathrm{B}}(1) \\
9^{\alpha \beta \mathrm{R}}(3)\end{array}$ & $9^{\mathrm{R}}(1)$ & $9^{\alpha \beta}(1)$ & $\begin{array}{c}9(1), 9^{\alpha \mathrm{B}}(1), \\
9^{\alpha \beta}(1), 9^{\alpha \beta \mathrm{B}}(1), \\
9^{\beta \mathrm{B}}(2)\end{array}$ \\
\hline & $\begin{array}{c}10(5), 10^{\alpha}(3) \\
10^{\alpha \mathrm{BCG}}(1), 10^{\alpha \mathrm{BE}}(1)\end{array}$ & $10^{\alpha \mathrm{BE}}(1), 10^{\mathrm{B}}(2)$ & $10^{\mathrm{C}}(1)$ & $\begin{array}{c}10(3), 10^{\alpha}(3) \\
10^{\alpha \mathrm{C}}(1)\end{array}$ & $\begin{array}{c}10(13), 10^{\alpha}(8) \\
10^{\mathrm{C}}(1)\end{array}$ \\
\hline & $\begin{array}{c}11(2), 11^{\alpha \mathrm{BCE}}(1), \\
11^{\alpha \mathrm{DEGX}}(1), 11^{\mathrm{BC}}(1)\end{array}$ & $11^{\mathrm{E}}(7)$ & $11^{\mathrm{E}}(1)$ & $\begin{array}{c}11(26), 11^{\alpha}(5), \\
11^{\alpha \mathrm{E}}(1), 11^{\alpha \mathrm{EC}}(1), \\
11^{\beta}(6), 11^{\mathrm{BC}}(1), \\
11^{\mathrm{E}}(10)\end{array}$ & $\begin{array}{c}11(22), 11^{\alpha}(1) \\
11^{\alpha \mathrm{BEX}}(1), 11^{\alpha \mathrm{C}}(1) \\
11^{\mathrm{E}}(7)\end{array}$ \\
\hline & $\begin{array}{c}16^{\alpha}(1) \\
16^{\alpha \mathrm{BEG}}(1)\end{array}$ & $16^{\alpha \mathrm{BEG}}(1)$ & $16^{\alpha}(4), 16^{\alpha \mathrm{E}}(2)$ & $\begin{array}{c}16^{\alpha \mathrm{BE}}(3), 16^{\alpha \mathrm{D}}(1), \\
16^{\alpha \mathrm{E}}(6), 16^{\alpha \mathrm{EG}}(2), \\
16^{\alpha \mathrm{G}}(2), 16^{\mathrm{B}}(1), \\
16^{\mathrm{BE}}(1), 16^{\mathrm{G}}(2)\end{array}$ & $\begin{array}{c}16^{\alpha}(2), 16^{\alpha \mathrm{B}}(1), \\
16^{\alpha \mathrm{BE}}(5), 16^{\alpha \mathrm{BG}}(1), \\
16^{\alpha \mathrm{E}}(4), 16^{\mathrm{B}}(6), \\
16^{\mathrm{BE}}(3), 16^{\mathrm{BEG}}(1), \\
16^{\mathrm{E}}(4), 16^{\mathrm{EG}}(1), \\
16^{\mathrm{G}}(1)\end{array}$ \\
\hline & $\mathrm{U}^{\alpha}(1), \mathrm{U}^{\alpha \mathrm{EX}}(1)$ & $\begin{array}{c}\mathrm{U}^{\alpha \mathrm{B}}(1), \mathrm{U}^{\alpha \mathrm{B}}(1), \\
\mathrm{U}^{\alpha \beta \mathrm{EG}}(2), \mathrm{U}^{\alpha \mathrm{E}}(1), \\
\mathrm{U}^{\beta}(1), \mathrm{U}^{\beta \mathrm{B}}(1), \\
\mathrm{U}^{\beta \mathrm{E}}(1)\end{array}$ & $\begin{array}{c}\mathrm{U}(1), \mathrm{U}^{\alpha \beta \mathrm{B}}(1), \\
\mathrm{U}^{\alpha \beta}(5), \mathrm{U}^{\alpha \beta \mathrm{G}}(1), \\
\mathrm{U}^{\beta}(14), \mathrm{U}^{\beta \mathrm{R}}(1), \\
\mathrm{U}^{\mathrm{R}}(1)\end{array}$ & $\begin{array}{c}\mathrm{U}^{\alpha \beta}(2), \mathrm{U}^{\alpha \beta \mathrm{X}}(3), \\
\mathrm{U}^{\alpha \mathrm{CER}}(2), \mathrm{U}^{\alpha \mathrm{EX}}(1), \\
\mathrm{U}^{\alpha \mathrm{ERX}}(1), \mathrm{U}^{\beta \mathrm{BE}}(1), \\
\mathrm{U}^{\beta \mathrm{B}}(1), \mathrm{U}^{\beta \mathrm{BX}}(2), \\
\mathrm{U}^{\beta \mathrm{G}}(1), \mathrm{U}^{\mathrm{BEX}}(1), \\
\mathrm{U}^{E}(1)\end{array}$ & $\begin{array}{c}\mathrm{U}^{\alpha}(3), \mathrm{U}^{\alpha \beta \mathrm{B}}(5), \\
\mathrm{U}^{\alpha \beta \mathrm{BX}}(4), \mathrm{U}^{\alpha \beta \mathrm{GX}}(1), \\
\mathrm{U}^{\alpha \beta}(11), \mathrm{U}^{\alpha \beta \mathrm{G}}(1), \\
\mathrm{U}^{\alpha \beta \mathrm{X}}(3), \mathrm{U}^{\alpha \mathrm{E}}(2), \\
\mathrm{U}^{\alpha \mathrm{X}}(1), \mathrm{U}^{\beta}(2), \\
\mathrm{U}^{\beta \mathrm{BEX}}(4), \mathrm{U}^{\beta \mathrm{BX}}(1), \\
\mathrm{U}^{\beta \mathrm{EX}}(1), \mathrm{U}^{\mathrm{B}}(1), \\
\mathrm{U}^{\mathrm{BE}}(1), \mathrm{U}^{\mathrm{EX}}(2)\end{array}$ \\
\hline
\end{tabular}


TABle 2: Continued.

\begin{tabular}{|c|c|c|c|c|c|}
\hline \multirow{2}{*}{$\begin{array}{l}\text { Host species: } \\
\text { Interface }^{1}\end{array}$} & \multicolumn{2}{|c|}{ Bighorn sheep } & \multirow{2}{*}{$\begin{array}{l}\text { Goat } \\
\text { Yes }\end{array}$} & \multicolumn{2}{|c|}{ Domestic sheep } \\
\hline & No & Yes & & Yes & No \\
\hline No. of populations & 7 & 3 & 1 & 6 & 6 \\
\hline No. of animals: & 234 & 105 & 45 & 152 & 219 \\
\hline No. of Pasteurellaceae isolates & 144 & 121 & 203 & 389 & 401 \\
\hline \multicolumn{6}{|l|}{ Species } \\
\hline Pasteurella multocida & multocida a (1) & $\begin{array}{l}\text { multocida a (4), } \\
\text { biotype } \mathrm{U}^{6}(2)\end{array}$ & $\begin{array}{c}\text { multocida a }(2), \\
\text { biotype } \mathrm{U}^{16}(1), \\
\text { biotype } \mathrm{U}^{6}(3)\end{array}$ & $\begin{array}{c}\text { multocida a (7), } \\
\text { multocida b }(7), \\
\text { canis }(4), \\
\text { Septica }(35), \\
\text { biotype } \mathrm{U}^{6}(3)\end{array}$ & $\begin{array}{l}\text { multocida a }(1) \text {, } \\
\text { septica }(10), \\
\text { biotype } \mathrm{U}^{23}(1), \\
\text { biotype } \mathrm{U}^{24}(1)\end{array}$ \\
\hline \multirow[t]{8}{*}{$\begin{array}{l}\text { Pasteurella (Bibersteinia) } \\
\text { trehalosi }\end{array}$} & $2(32), 2^{\mathrm{B}}(53)$ & $2^{\mathrm{B}}(35), 2^{\mathrm{BCDE}}(1)$, & $2(25), 2^{\mathrm{C}}(7)$ & $2(53), 2^{\mathrm{B}}(1)$ & $2(63), 2^{\mathrm{BCDE}}(1)$, \\
\hline & $2^{\mathrm{BC}}(1), 2^{\mathrm{BCE}}(1)$ & $2^{\mathrm{BD}}(4), 2^{\mathrm{BE}}(11)$ & $2^{\mathrm{CD}}(1), 2^{\mathrm{CDS}}(1)$, & $2^{\mathrm{C}}(2), 2^{\mathrm{CDES}}(2)$, & $2^{\mathrm{C}}(7), 2^{\mathrm{CDE}}(1)$ \\
\hline & $2^{\mathrm{BD}}(1), 2^{\mathrm{BE}}(12)$, & $2^{\mathrm{BG}}(4), 2^{\mathrm{BS}}(13)$ & $2^{\mathrm{CE}}(1), 2^{\mathrm{E}}(62)$ & $2^{\mathrm{CDS}}(2), 2^{\mathrm{CE}}(1)$ & $2^{\mathrm{CDES}}(2), 2^{\mathrm{CDS}}(1)$ \\
\hline & $2^{\mathrm{BGS}}(1), 2^{\mathrm{C}}(1)$ & $2^{\mathrm{C}}(1), 2^{\mathrm{E}}(3)$ & & $2^{\mathrm{CES}}(1), 2^{\mathrm{CS}}(2)$ & $2^{\mathrm{CE}}(1), 2^{\mathrm{DES}}(1)$ \\
\hline & $2^{\mathrm{CD}}(1), 2^{\mathrm{CDS}}(1)$ & $2^{\mathrm{G}}(3), 2^{\mathrm{GS}}(1)$ & & $2^{\mathrm{E}}(5), 2^{\mathrm{EG}}(1)$ & $2^{\mathrm{E}}(2), 2^{\mathrm{EG}}(1)$ \\
\hline & $2^{\mathrm{E}}(2), 2^{\mathrm{EG}}(1), 2^{\mathrm{GS}}(1)$ & $2^{S}(4)$ & & $\begin{array}{c}2^{\mathrm{EGS}}(1), 2^{\mathrm{ES}}(1) \\
2^{\mathrm{G}}(1), 2^{\mathrm{GS}}(1) \\
2^{\mathrm{S}}(4)\end{array}$ & $2^{\mathrm{G}}(4), 2^{\mathrm{S}}(1)$ \\
\hline & $\begin{array}{c}4^{\mathrm{B}}(3), 4^{\mathrm{BDS}}(1), \\
4^{\mathrm{CDS}}(1)\end{array}$ & & $4^{\mathrm{CDS}}(5)$ & $\begin{array}{c}4(1), 4^{\mathrm{CDES}}(2), \\
4^{\mathrm{CDS}}(14), 4^{\mathrm{G}}(1)\end{array}$ & $\begin{array}{c}4(1), 4^{\mathrm{BCDS}}(1), \\
4^{\mathrm{CD}}(1), 4^{\mathrm{CDE}}(3),\end{array}$ \\
\hline & & & & & $\begin{array}{c}4^{\mathrm{CDEGS}}(1), 4^{\mathrm{CDES}}(6), \\
4^{\mathrm{CDS}}(4)\end{array}$ \\
\hline
\end{tabular}

${ }^{1}$ Yes: $\leq 14.5 \mathrm{~km}$ to sympatric species; No: $>14.5 \mathrm{~km}$ to sympatric species or surrounded by development that prevents interspecific interactions.

twice had complete concordance in the biovariants identified for each sampling period. Among the domestic sheep $(n=$ $493)$ and goat $(n=219)$ isolates that were identified in both sampling periods, only $4 \%$ of sheep $(n=20)$ and goat $(n=9)$ isolates were identified at both sample events from the same individual. Two isolates were identified in the same domestic sheep during both sample periods on two occasions.

3.3. Bacteriology: Mycoplasma. Swabs from bighorn sheep $(n=248)$, domestic sheep $(n=166)$, and domestic goat ( $n=18$ ) were cultured for Mycoplasma. Mycoplasma was isolated from $60-100 \%$ of sampled individuals in each of the domestic livestock populations, with the exception of one domestic sheep population without Mycoplasma isolates from 13 sampled animals. In contrast, Mycoplasma was not isolated from any of the bighorn sheep sampled.

3.4. Bacteriology: Euthanized Bighorn Sheep. A male bighorn sheep euthanized for closely associated with domestic sheep and goats had no apparent clinical disease. Two biovariants P. (B.) trehalosi $2^{\mathrm{CDS}}$ and $P$. (B.) trehalosi $4^{\mathrm{CDS}}$ ) isolated from this male were not identified in the closest bighorn sheep population, but were identified in the sympatric domestic livestock. Pasteurella (B.) trehalosi $2^{\mathrm{B}}$, which was the most common biovariant isolated from bighorn sheep $(n=88)$, was isolated from the euthanized male and was identified in one sympatric domestic sheep. Pasteurella (B.) trehalosi 2, Bacillus sp., and Arcanobacterium pyogenes were also isolated from the bighorn sheep male and sympatric livestock. Samples from this male were negative for Mycoplasma sp., although Mycoplasma sp. was isolated from the sympatric goat and domestic sheep population.

3.5. Virology. Every population tested had serologic evidence of PI-3 virus (Table 3). All populations except for some isolated populations of domestic sheep $(n=1)$ and bighorn sheep $(n=3)$ had serologic evidence for BRSV. Five individuals in two domestic sheep populations had serologic evidence for both BVDV-1 and BVDV-2, and all titers were $<1: 128$. Two bighorn sheep and one goat had low titers $(1: 8)$ to IBR, and the goat was at the same interface as one of the bighorn sheep. Of the domestic sheep $(n=85)$ in three populations and domestic goats $(n=34)$ in one population that were sampled six months apart, there was evidence for seroconversion to PI-3 $(n=26)$ and $\operatorname{BRSV}(n=5)$. For domestic sheep with signs of respiratory disease $(n=11)$, there was evidence for antibodies to PI-3 $(n=9)$ and BRSV $(n=5)$, but not BVDV-1, BVDV-2, or IBR.

3.6. Parasitology. Fecal samples were evaluated for isolated $(n=165)$ and interface $(n=98)$ bighorn sheep among six isolated and three interface populations. Fecal samples were evaluated for isolated $(n=36)$ and interface 
TABle 3: Number (\%) of bighorn sheep, domestic sheep, and domestic goats with serologic evidence for antibodies to parainfluenza-3, bovine respiratory syncytial virus, bovine viral diarrhea-1 and bovine viral diarrhea-2, and infectious bovine rhinotracheitis in isolated and interface populations.

\begin{tabular}{|c|c|c|c|c|c|}
\hline \multirow{2}{*}{$\begin{array}{l}\text { Species } \\
\text { Location }\end{array}$} & \multicolumn{2}{|c|}{ Bighorn } & \multirow{2}{*}{$\begin{array}{l}\text { Domestic goat } \\
\text { Interface }\end{array}$} & \multicolumn{2}{|c|}{ Domestic sheep } \\
\hline & Isolated & Interface & & Interface & Isolated \\
\hline No. of populations & 7 & 3 & 1 & 6 & 6 \\
\hline No. of animals tested & 198 & 105 & 44 & 143 & 214 \\
\hline Parainfluenza-3 & $165(83 \%)$ & $91(87 \%)$ & $9(20 \%)$ & $102(71 \%)$ & $113(53 \%)$ \\
\hline Bovine respiratory syncytial virus & $57(29 \%)$ & $76(72 \%)$ & $44(100 \%)$ & $95(66 \%)$ & $104(49 \%)$ \\
\hline Bovine viral diarrhea-1 & $0(0 \%)$ & $0(0 \%)$ & $0(0 \%)$ & $1(0.7 \%)$ & $3(1 \%)$ \\
\hline Bovine viral diarrhea-2 & $1(0.5 \%)$ & $0(0 \%)$ & $0(0 \%)$ & $1(0.7 \%)$ & $6(3 \%)$ \\
\hline Infectious bovine rhinotracheitis & $0(0 \%)$ & $2(2 \%)$ & $1(2 \%)$ & $0(0 \%)$ & $0(0 \%)$ \\
\hline
\end{tabular}

$(n=44)$ domestic sheep among three isolated and six interface populations. Twelve fecal samples were analyzed from the goat population. Protostrongylus and Dictyocaulus sp. were identified in isolated and interface bighorn sheep populations, whereas dorsal-spined larvae presumed to be Muellerius sp. were identified in an isolated bighorn sheep population. Representatives of Trichostongylina, Moniezia spp., Strongyloides sp., and Eimeria sp. were identified in the goat population. Trichostongylina, Moniezia spp., and Eimeria sp. were identified in isolated and interface domestic sheep populations, whereas Strongyloides sp. and Dictyocaulus sp. were identified in interface domestic sheep populations. Muellerius sp. was not identified in domestic goat and sheep populations. Livestock were treated with anthelmintics at least once during the previous year.

\section{Discussion}

This study documented the presence of multiple bacterial, viral, and parasite species in bighorn sheep and livestock populations distant from and at the domestic animal/wildlife interface. Although the sites where these populations were sampled do not reflect the extensive translocations of bighorn sheep that have been conducted for management purposes or individual animal movements between populations [3], these results provide important baseline data for understanding agents potentially responsible for respiratory disease in bighorn and domestic sheep under field conditions. Populations of varying size (Table 1) were sampled opportunistically based on agency or collaborator activities with bighorn sheep, and livestock operator's willingness to participate. Consequently, based on standards for observational studies [41], the limitations of extrapolating inferences from this study to other populations and locations must be acknowledged. Also, few males were sampled in this study, and all wildlife and domestic populations at the interfaces studied could not be sampled.

4.1. Populations Studied. Sampled bighorn sheep populations varied by an order of magnitude in estimated size and density (Table 1). Domestic sheep populations varied by two orders of magnitude and ranged from small family operations to large, open range populations. While bighorn sheep were exclusively on public land (federal and state), domestic livestock were largely on private land. This finding is, in part, a function of the populations sampled for this study. However, it illustrates that, while there has been contention over domestic livestock grazing on public lands $[42,43]$, the potential for conflicting management objectives also exists where domestic livestock are on private land near to public lands that are populated with bighorn sheep. The range in sizes of sampled bighorn and domestic sheep populations met the objective of establishing representative baseline data, although further research is needed to establish whether there are patterns associated with population size.

4.2. Bacteriology: Pasteurellaceae. Pasteurellaceae are a heterogeneous mix of many bacterial strains that can cause a range of clinical signs [44]. For this study, an established biovariant classification scheme was used for Pasteurellaceae isolates because it offered the best opportunity to classify isolates and provide useful epidemiological data. Collection of swabs for bacteriological cultures was conducted according to standardized sampling and laboratory procedures developed by the Western Wildlife Health Committee, Association of Western Fish and Wildlife Agencies [28].

Pasteurellaceae associated with respiratory disease in bighorn and domestic sheep are M. (Pasteurella) haemolytica, P. (B.) trehalosi (formerly P. haemolytica biotype T), and P. multocida [44-50]. These bacteria are also found in animals without disease, whether serotype or biovariant subclassification schemes are used to characterize isolates $[33,51-54]$. Under in vivo and in vitro experimental conditions, bighorn sheep appear to be more susceptible to disease due to $M$. haemolytica than are domestic sheep and other species $[17,18,55,56]$. It is uncertain whether a similar etiopathogenesis occurs with other Pasteurallaceae species and how experimental results can be applied to predicting and mitigating respiratory disease outbreaks in free-ranging populations.

Multiple Pasteurellaceae species or biovariants were identified in the host species in this study, and most biovariants were represented by only a few isolates (Table 2). While $M$. haemolytica and P. multocida were primarily associated with domestic sheep and $P$. (B.) trehalosi comprised most bighorn sheep isolates, Pasteurellaceae biovariants often occurred 
among multiple host species, and there was a complex assemblage of Pasteurellaceae and non-Pasteurellaceae species [57]. This is similar to results of previous studies in Nevada and California that used biovariant and serotype classification schemes, and it presents challenges for identifying patterns and making rigorous inferences $[53,54]$. These challenges are expanded by the overlap of isolates identified in this study among largely healthy animals compared with retrospective studies of domestic and bighorn sheep with respiratory disease $[58,59]$.

The cross-sectional study design and the dearth of animals with clinical signs of disease preclude identification of pathogenic Pasteurellaceae or the potential for cross-species transmission to result in disease, but data is presented that is germane to these topics. There was no evidence for an increased risk for beta-hemolytic or "unique" biovariants to be identified in interface populations. This suggests that bighorn and domestic sheep may be colonized by leukotoxinpositive Pasteurellaceae without the development of disease, although further work is needed to clarify whether the numerous biovariants and their occurrence in multiple host species could easily obscure cross-species transmission of any Pasteurellaceae that might have occurred. Similarly, the observation of a single, apparently healthy bighorn sheep ram that shared shelter, food, and water with domestic sheep and goats for $>6$ mo has limited inference regarding cross-species transmission in either direction. However, the identification of biovariants that were not found in other members of the same species in the ram and one domestic sheep is consistent with, but not definitive for, cross-species transmission. Further study using DNA fingerprinting or sequencing technology would be needed to confirm the similarity of these isolates but would not confirm direction of transmission without longitudinal data. Nevertheless, these data support removal of individuals that associate with sympatric species where there is a low tolerance for possible interspecies transmission of agents.

Pasteurellaceae results from individual livestock resampled 6 mo apart were compared as a means of assessing temporal variation (Table 3 ). Minimal concordance in culture results for these individuals suggests that oropharyngeal microflora may be temporally dynamic, that sample numbers or swabbing methods may be inadequate to characterize the great diversity of biovariants that are present, or that competition among biovariants in culture or standard microbial culture procedures are responsible for this variation. Regardless of the reasons for these results, single sampling events for domestic livestock may not be appropriate for some research and management questions. A previous study similarly suggests that bighorn sheep Pasteurellaceae may vary temporally, but results were confounded by use of antibiotics [34]. It is unlikely that this variation is due to inconsistent classification of isolates, because there is substantial consistency in assigned Pasteurellaceae biovariant classifications among isolates that are recharacterized as a part of routine reference bank and quality control procedures.

The diversity of Pasteurellaceae observed in this study and others presents challenges for interpretation. The observation of many, uncommonly identified biovariants in this study indicate that more extensive sampling is required to fully characterize the Pasteurellaceae of bighorn sheep and sympatric livestock. Additional data from animals with respiratory disease is needed to determine the pathogenicity of biovariants, as well as the impact upon populations. Temporal variation or inconsistent detection of Pasteurellaceae suggests the need to develop population level sampling strategies and interpretations of agent health impacts [60]. Although there has been extensive research on Pasteurellaceae biovariants and their pathogenesis in bighorn sheep, future work that characterizes risk factors for individuals and that compares populations may yield data that is useful for management purposes.

4.3. Bacteriology: Mycoplasma sp. Mycoplasma ovipneumoniae has been associated with respiratory disease as a primary pathogen of small ruminants and may increase their susceptibility to secondary pasteurellosis, particularly in lambs 2-12 months of age [61, 62]. This could explain reduced lamb recruitment following bighorn sheep outbreaks [48, 62, 63]. However, undifferentiated Mycoplasma was isolated from apparently healthy animals in all but one domestic livestock population in this study. This is similar to previous studies that suggest that M. ovipneumoniae and possibly other Mycoplasma species may be common respiratory tract commensals that only cause disease in animals that are compromised due to other causes [64].

In contrast to domestic sheep, bighorn sheep populations in this study appeared to be naïve to Mycoplasma. This naivety suggests the potential for an outbreak if this agent was introduced. Alternatively, although the species of Mycoplasma was not established, Mycoplasma may not be easily transmitted to bighorn sheep. Limited inference to support this is the failure to isolate Mycoplasma from the single male bighorn sheep that was closely associated with livestock infected with Mycoplasma.

Whether Mycoplasma can be an opportunistic or primary pathogen in free-ranging bighorn sheep is not clear. While a high degree of association between M. ovipneumoniae and bronchopneumonia in free-ranging bighorn sheep has been reported, the percentage of free-ranging pneumonic bighorn with culture or PCR evidence of Mycoplasma infection varies from 7 to $55 \%[23,36,48]$. In addition, limited experimental inoculations in bighorn sheep lambs suggest that $M$. ovipneumoniae infection alone is insufficient to cause fatal respiratory disease [23]. This variation could be due to methodological limitations [23], variation in the agents responsible for different outbreaks, or other reasons. In particular, it is important to recognize that Mycoplasma sp. is not routinely isolated with general microbiological methods that are routinely employed by diagnostic laboratories. While this study employed methods developed for Mycoplasma sp. by a laboratory with expertise in Mycoplasma sp. isolation, there is a need for additional research to clarify methods and sample sizes that are required for strong inferences from research on bighorn and domestic sheep populations. 
4.4. Virology. The viral respiratory agents in this study were selected based on their potential to cause respiratory disease or predispose to pneumonic pasteurellosis in domestic and wild ruminants [22, 65-67]. A high percentage of the domestic livestock and bighorn sheep in this study had evidence of antibodies to PI-3 and BRSV (Table 3). There was evidence of seroconversion for BRSV and PI-3 among domestic sheep and goats that were sampled twice. Parainfluenza-3 and BRSV (or reported as RSV) have been associated with respiratory disease in bighorn sheep, and domestic sheep and goats $[36,65,68,69]$. However, antibodies to these agents were also present in apparently healthy animals in these references and others $[22,36,70-72]$. This indicates that survival from infections is possible and perhaps probable in populations with high serologic prevalence.

In contrast to PI-3 and BRSV, few animals had evidence of antibodies to BVDV-1, BVDV-2, or IBR (Table 3). Limited documentation exists on the clinical effect of these infections in domestic sheep and goats $[68,69,73]$. Serologic evidence of BVDV and IBR infections in healthy bighorn sheep indicates that these animals can survive infections with these agents [22, 70]. However, isolation of IBR from 3 of 6 lung samples from bighorn sheep during a Tendoys, Montana outbreak, isolation of BVDV from 14 of 19 bighorn sheep lungs during a Lost Creek, Montana outbreak [22], and $>$ fourfold increases in serologic titers to BVDV during the Hells Canyon outbreak [36] suggest a potential role for these viruses in some bighorn sheep die-offs. Based on domestic ruminant models, these viruses may cause primary infections that result in secondary, opportunistic pneumonic pasteurellosis under some conditions or be non- to mildly pathogenic in other circumstances $[36,65,74]$. Further research is needed to clarify the degree, frequency, and circumstances under which these agents pose a risk for causing disease.

4.5. Parasitology. Evaluations of parasitic infections based on fecal analyses (helminth larvae and eggs, oocysts) are included in this study due to the potential for gastrointestinal parasites to predispose animals to disease from other agents and the role of lungworms in ungulate respiratory disease $[12,67,75]$. Only presence and absence data are reported herein as validated and standardized, and quantitative methods for assessing parasite numbers were not available for this study. The nematode, cestode, and protozoan parasites identified are similar to those previously reported for domestic and bighorn sheep, and their impact on the populations studied are uncertain [75-77]. Muellerius sp. is generally associated with domestic sheep, rather than bighorn sheep [78]. Evidence for Muellerius sp. in an isolated bighorn sheep population may represent historic introduction and establishment of this parasite in this bighorn sheep population, or unidentified recent introduction [79]. Additionally, it has become apparent that not all reports of dorsal-spined larvae may be attributable to Muellerius but could represent another protostrongylid muscleworm, Parelaphostrongylus odocoilei known to infect bighorn at some localities $[79,80]$. Across this assemblage of free-ranging and domestic host species, historic introductions in other settings are thought to be responsible for a mosaic landscape of native and introduced parasite species [81]. This suggests the potential for a similar mosaic faunal structure for bacterial and viral agents, as a consequence of historic transmission events across the bighorn/domestic sheep interface, which could account from some of the results in this study.

\section{Conclusions}

This study documents the presence of multiple Pasteurellaceae biovariants, Mycoplasma, and viruses in apparently healthy bighorn sheep, domestic sheep, and goats that are at the domestic animal/wildlife interface and in isolated populations. When these results are considered with their presence in animals with respiratory disease in other reports $[22,23,36,58,59]$, it is evident that further work is needed to clarify environmental, agent, and host determinants of respiratory disease, as well as standardize sampling and laboratory procedures. Clarification of whether these agents are primary pathogens, secondary pathogens, commensals, or predispose to outbreaks due to other agents is important.

Given the polarized debate over management practices at the bighorn/domestic sheep interface, the potential for results of this study to be selectively interpreted exists. Reflecting upon basic animal disease control principles and how they might be applied to free-ranging wildlife will be more useful. Any time contact occurs among populations, the potential for transmission of novel agents to naïve animals exists [82], and there are established quarantine, vaccination, testing, risk assessment, and other strategies for minimizing spread of infectious diseases among translocated domestic and wild animal populations [83-85]. However, there is a need to rigorously document the true risks of interspecies transmission under field conditions, as well as determine the efficacy of different management strategies. Strategies may vary by circumstances, as management of small or otherwise highly valued bighorn sheep populations may result in adoption of risk-averse strategies. Similarly, domestic sheep operations that are considered critical for a local economy, for exotic weed control, to prevent conversion of land to uses that are not compatible with wildlife or agricultural interests, or for other reasons, may require management strategies that protect their interests. For all other situations, management will be guided by sociological values and biological perceptions until the biological risks and options are clarified, and a sociologically based structure for decision making is agreed upon.

\section{Acknowledgments}

The authors thank Becky Frye and all other individuals who assisted with collection of domestic livestock samples, the capture of bighorn sheep, and the collection and submission of samples, as well as the many individuals that contributed to the development of the project. This research was supported in part by funds provided by the University of Idaho, College of Agriculture and Life Sciences, Montana Fish Wildlife and Parks, the Foundation for North American Wild Sheep, Idaho Department of Fish and Game, Montana 
State University, and Program of Economically Important Infectious Animal Diseases the Colorado State University. They thank E. P. Hoberg, P. J. P. Gogan; J. G. Powers, and anonymous reviewers for their helpful comments on earlier drafts of this paper. Any use of trade, product, or firm names is for descriptive purposes only and does not imply endorsement by the U.S. Government.

\section{References}

[1] H. K. Buechner, "The bighorn sheep in the United States, its past, present, and future," Wildlife Monographs, vol. 4, pp. 1$174,1960$.

[2] J. E. Gross, F. J. Singer, M. E. Moses, and P. R. Krausman, "Effects of disease, dispersal, and area on bighorn sheep restoration," Restoration Ecology, vol. 8, no. 4, pp. 25-37, 2000.

[3] D. E. Toweill and V. Geist, Return of Royalty: Wild Sheep of North America, Boone and Crockett Club and Foundation for North American Wild Sheep, Missoula, Mont, USA, 1999.

[4] W. T. Hornaday, "Notes on the mountain sheep of North America with a description of a new species," New York Zoological Society Annual Report, vol. 5, pp. 77-122, 1901.

[5] W. A. Baillie-Grohman, "Camps on the trail of the bighorn," in Camps in the Rockies, W. A. Baillie-Grohman, Ed., pp. 154181, Charles Scribner's Sons, New York, NY, USA, 1902.

[6] W. M. Rush, "Notes on disease in wild game," Journal of Mammalogy, vol. 8, no. 2, pp. 163-164, 1927.

[7] H. F. Evans, "Bighorn at many glacier," Glacial Drift, vol. 10, no. 1, pp. 2-3, 1937.

[8] H. B. Mills, "A preliminary study of the bighorn of Yellowstone National Park," Journal of Mammalogy, vol. 18, pp. 205-212, 1937.

[9] H Marsh, "Pneumonia in Rocky Mountain bighorn sheep," Journal of Mammalogy, vol. 19, no. 2, pp. 214-219, 1938.

[10] M. K. Potts, "Hemorrhagic septicemia in the bighorn of Rocky Mountain National Park," Journal of Mammalogy, vol. 18, pp. 105-106, 1937.

[11] M. W. Miller, N. T. Hobbs, and E. S. Williams, "Spontaneous pasteurellosis in captive Rocky Mountain bighorn sheep (Ovis canadensis canadensis): clinical, laboratory, and epizootiological observations," Journal of Wildlife Diseases, vol. 27, no. 4, pp. 534-542, 1991.

[12] D. J. Forrester and C. M. Senger, "A survey of lungworm infection in bighorn sheep of Montana," Journal of Wildlife Management, vol. 28, no. 3, pp. 481-491, 1964.

[13] W. J. Foreyt and D. A. Jessup, "Fatal pneumonia of bighorn sheep following association with domestic sheep," Journal of Wildlife Diseases, vol. 18, no. 2, pp. 163-168, 1982.

[14] D. K. Onderka and W. D. Wishart, "Experimental contact transmission of Pasteurella haemolytica from clinically normal domestic sheep causing pneumonia in Rocky Mountain bighorn sheep," Journal of Wildlife Diseases, vol. 24, no. 4, pp. 663-667, 1988.

[15] W. Foreyt, "Effects of controlled contact exposure between healthy bighorn sheep and llamas, domestic goats, mountain goats, cattle, domestic sheep, or mouflon sheep," in Biennial Symposium of the Northern Wild Sheep and Goat Council, pp. 7-14, Northern Wild Sheep and Goat Council, 1994.

[16] R. P. Dassanayake, S. Shanthalingam, C. N. Herndon et al., "Mannheimia haemolytica serotype Al exhibits differential pathogenicity in two related species, Ovis canadensis and Ovis aries," Veterinary Microbiology, vol. 133, no. 4, pp. 366-371, 2009.
[17] B. J. Kraabel and M. W. Miller, "Effect of simulated stress on susceptibility of bighorn sheep neutrophils to Pasteurella haemolytica leukotoxin," Journal of Wildlife Diseases, vol. 33, no. 3, pp. 558-566, 1997.

[18] P. K. Lawrence, S. Shanthalingam, R. P. Dassanayake et al., "Transmission of Mannheimia haemolytica from domestic sheep (Ovis aries) to bighorn sheep (Ovis canadensis): unequivocal demonstration with green fluorescent proteintagged organisms," Journal of Wildlife Diseases, vol. 46, no. 3, pp. 706-717, 2010.

[19] T. R. Spraker, C. P. Hibler, G. G. Schoonveld, and W. S. Adney, "Pathologic changes and microorganisms found in bighorn sheep during a stress-related die-off," Journal of Wildlife Diseases, vol. 20, no. 4, pp. 319-327, 1984.

[20] R. Dean, P. Hnilicka, T. J. Kreeger, and T. Delcurto, "An investigation into the selenium requirement for Rocky Mountain bighorn sheep," in Biennial Symposium of the Northern Wild Sheep and Goat Council, pp. 95-99, Northern Wild Sheep and Goat Council, 2002.

[21] P. Hnilicka, J. Mioncznski, B. J. Mincher et al., "Bighorn sheep lamb survival, trace minerals, rainfall, and air pollution: are there any connections?" in Biennial Symposium of the Northern Wild Sheep and Goat Council, pp. 69-94, Northern Wild Sheep and Goat Council, 2002.

[22] A. Aune, N. Anderson, D. E. Worley, L. Stackhouse, J. Henderson, and J. Daniel, "A comparison of population and health histories among seven Montana bighorn sheep populations," in Biennial Symposium of the Northern Wild Sheep and Goat Council, pp. 46-69, Northern Wild Sheep and Goat Council, 1998.

[23] T. E. Besser, E. F. Cassirer, K. A. Potter et al., "Association of Mycoplasma ovipneumoniae infection with populationlimiting respiratory disease in free-ranging Rocky Mountain bighorn sheep (Ovis canadensis canadensis)," Journal of Clinical Microbiology, vol. 46, no. 2, pp. 423-430, 2008.

[24] T. R. Spraker and C. P. Hibler, "Summer lamb mortality of Rocky Mountain bighorn sheep," in Biennial Symposium of the Northern Wild Sheep and Goat Council, pp. 11-12, Northern Wild Sheep and Goat Council, 1977.

[25] Unites States Department of the Interior, Bureau of Land Management, "Revised guidelines for management of somestic sheep and goats in native wild sheep habitats," Instruction Memorandum 98-140, 1998.

[26] Montana Fish Wildlife and Parks, "Montana Bighorn Sheep Conservation Strategy," Montana Fish, Wildlife \& Parks, Helena, MT, 2010, http://fwp.mt.gov/wildthings/manage ment/bighorn/plan.html.

[27] M. D. Kock, D. A. Jessup, R. K. Clark, C. E. Franti, and R. A. Weaver, "Capture methods in five subspecies of free-ranging bighorn sheep: an evaluation of drop-net, drive-net, chemical immobilization and the net-gun," Journal of Wildlife Diseases, vol. 23, no. 4, pp. 634-640, 1987.

[28] M. L. Drew, C. Gillin, and G. C. Weiser, Recommendations for Isolation of Pasteurella spp. and Mycoplasma spp. from Bighorn Sheep, Western Wildlife Health Committee, Association of Western Fish and Wildlife Agencies, 2005.

[29] R. D. Beane and N. T. Hobbs, "The Baermann technique for estimating Protostrongylus infection in bighorn sheep: effect of laboratory procedures," Journal of Wildlife Diseases, vol. 19, no. 1, pp. 7-9, 1983.

[30] K. L. Hoar, Parasite loads and their relationship to herd health in the Highlands bighorn sheep herd in southwestern Montana, M.S. thesis, Montana State University, Bozeman, Mont, USA, 1995. 
[31] B. V. Beaver, W. Reed, S. Leary et al., "2000 Report of the AVMA panel on euthanasia," Journal of the American Veterinary Medical Association, vol. 218, no. 5, pp. 669-696, 2001.

[32] M. D. Jaworski, A. C. S. Ward, D. L. Hunter, and I. V. Wesley, "Use of DNA analysis of Pasteurella haemolytica biotype T isolates to monitor transmission in bighorn sheep (Ovis canadensis canadensis)," Journal of Clinical Microbiology, vol. 31, no. 4, pp. 831-835, 1993.

[33] M. D. Jaworski, D. L. Hunter, and A. C. S. Ward, "Biovariants of isolates of Pasteurella from domestic and wild ruminants," Journal of Veterinary Diagnostic Investigation, vol. 10, no. 1, pp. 49-55, 1998.

[34] G. C. Weiser, D. S. Miller, M. L. Drew, J. C. Rhyan, and A. C. S. Ward, "Variation in pasteurella (bibersteinia) and mannheimia SPP. following transport and antibiotic treatment in free-ranging and captive rocky mountain bighorn sheep (Ovis canadensis canadensis)," Journal of Zoo and Wildlife Medicine, vol. 40, no. 1, pp. 117-125, 2009.

[35] R. M. Atlas, Handbook of Microbiological Media, CRC Press, Boca Raton, Fla, USA, 1993.

[36] K. M. Rudolph, D. L. Hunter, R. B. Rimler et al., "Microorganisms associated with a pneumonic epizootic in Rocky Mountain bighorn sheep (Ovis canadensis canadensis)," Journal of Zoo and Wildlife Medicine, vol. 38, no. 4, pp. 548-558, 2007.

[37] G. E. Cottral, Manual of Standardized Methods for Veterinary Microbiology, Cornell University Press, Ithaca, NY, USA, 1978.

[38] F. J. Fenner, E. P. G. Gibbs, F. Murphy, R. Rott, M. J. Studdert, and D. O. White, Veterinary Virology, Academic Press, San Diego, Calif, USA, 2nd edition, 1993.

[39] Center for Veterinary Biologics \& National Veterinary Service Laboratories, "Testing Protocol," Revision BPRRO2105.02, Ames, Iowa, USA, 1998.

[40] G. J. Tortora, B. R. Funke, and C. L. Case, Microbiology: An Introduction, The Benjamin/Cummings Publishing, Menlo Park, Calif, USA, 4th edition, 1992.

[41] I. Dohoo, W. Martin, and H. Stryhn, Veterinary Epidemiologic Research, AVC, Charlottetown, Prince Edward Island, Canada, 2003.

[42] United States Geologic Survey/Bureau of Reclamation Office, "Payette National Forest Science Panel Discussion on risk for disease transmission analysis between bighorn and domestic sheep," Tech. Rep., United States Geologic Survey/Bureau of Reclamation Office, Boise, Idaho, USA, 2006.

[43] Council for Agricultural Science and Technology (CAST), "Pasteurellosis transmission risks between domestic and wild sheep," CAST Commentary QTA2008-1, CAST, Ames, Iowa, USA, 2008.

[44] M. W. Miller, "Pasteurellosis," in Infectious Diseases of Wild Mammals, E. S. Williams and I. K. Barker, Eds., pp. 330-349, Iowa State University Press, Ames, Iowa, USA, 3rd edition, 2001.

[45] O. Angen, R. Mutters, D. A. Caugant, J. E. Olsen, and M. Bisgaard, "Taxonomic relationships of the [Pasteurella] haemolytica complex as evaluated by DNA-DNA hybridizations and 16s rRNA sequencing with proposal of Mannheimia haemolytica gen. nov., comb. nov., Mannheimia granulomatis comb. nov., Mannheimia glucosida sp. nov., Mannheimia ruminalis sp. nov. and Mannheimia varigena sp. nov," International Journal of Systematic Bacteriology, vol. 49, no. 1, pp. 67-86, 1999.

[46] P. J. Watson and R. L. Davies, "Outbreak of Pasteurella multocida septicaemia in neonatal lambs," Veterinary Record, vol. 151, no. 14, pp. 420-422, 2002.
[47] G. C. Weiser, W. J. DeLong, J. L. Paz, B. Shafii, W. J. Price, and A. C. S. Ward, "Characterization of Pasteurella multocida associated with pneumonia in bighorn sheep," Journal of Wildlife Diseases, vol. 39, no. 3, pp. 536-544, 2003.

[48] J. L. George, D. J. Martin, P. M. Lukacs, and M. W. Miller, "Epidemic pasteurellosis in a bighorn sheep population coinciding with the appearance of a domestic sheep," Journal of Wildlife Diseases, vol. 44, no. 2, pp. 388-403, 2008.

[49] P. J. Blackall, A. M. Bojesen, H. Christensen, and M. Bisgaard, "Reclassification of [Pasteurella] trehalosi as Bibersteinia trehalosi gen. nov., comb. nov," International Journal of Systematic and Evolutionary Microbiology, vol. 57, no. 4, pp. 666-674, 2007.

[50] P. H. A. Sneath and M. Stevens, “Actinobacillus rossii sp. nov., Actinobacillus seminis sp. nov., nom. rev., Pasteurella bettii sp. nov., Pasteurella lymphangitidis sp. nov., Pasteurella mairi sp. nov., and Pasteurella trehalosi sp. nov," International Journal of Systematic Bacteriology, vol. 40, no. 2, pp. 148-153, 1990.

[51] M. R. Dunbar, A. C. Ward, and G. Power, "Isolation of Pasteurella haemolytica from tonsillar biopsies of Rocky Mountain bighorn sheep," Journal of Wildlife Diseases, vol. 26, no. 2, pp. 210-213, 1990.

[52] N. J. L. Gilmour, D. A. Thompson, and J. Fraser, "The recovery of Pasteurella haemolytica from the tonsils of adult sheep," Research in Veterinary Science, vol. 17, no. 3, pp. 413-414, 1974.

[53] A. C. S. Ward, D. L. Hunter, M. D. Jaworski et al., "Pasteurella spp. in sympatric bighorn and domestic sheep," Journal of Wildlife Diseases, vol. 33, no. 3, pp. 544-557, 1997.

[54] L. Tomassini, B. Gonzales, G. C. Weiser, and W. Sischo, "An ecologic study comparing distribution of Pasteurella trehalosi and Mannheimia haemolytica between Sierra Nevada bighorn sheep, White Mountain bighorn sheep, and domestic sheep," Journal of Wildlife Diseases, vol. 45, no. 4, pp. 930-940, 2009.

[55] D. K. Onderka, S. A. Rawluk, and W. D. Wishart, "Susceptibility of Rocky Mountain bighorn sheep and domestic sheep to pneumonia induced by bighorn and domestic livestock strains of Pasteurella haemolytica," Canadian Journal of Veterinary Research, vol. 52, no. 4, pp. 439-444, 1988.

[56] R. P. Dassanayake, W. Liu, W. C. Davis, W. J. Foreyt, and S. Srikumaran, "Bighorn sheep $\beta 2$-integrin LFA-1 serves as a receptor for Mannheimia haemolytica leukotoxin," Journal of Wildlife Diseases, vol. 44, no. 3, pp. 743-747, 2008.

[57] S. Safaee, G. C. Weiser, E. F. Cassirer, R. R. Ramey, and S. T. Kelley, "Microbial diversity in bighorn sheep revealed by culture-independent methods," Journal of Wildlife Diseases, vol. 42, no. 3, pp. 545-555, 2006.

[58] D. S. Miller, G. C. Weiser, A. C. Ward, M. L. Drew, and P. L. Chapman, "Bighorn sheep Pasteurellaceae isolates from submissions to the Caine Veterinary Teaching Center (19892004)," American Journal of Veterinary Research. In press.

[59] D. S. Miller, G. C. Weiser, A. C. Ward, M. L. Drew, and P. L. Chapman, "Domestic sheep (Ovis aries) Pasteurellaceae isolates from submissions to the Caine Veterinary Teaching Center (1990-2004)," Veterinary Microbiology, vol. 150, no. 34, pp. 284-288, 2011.

[60] M. T. Collins, I. A. Gardner, F. B. Garry, A. J. Roussel, and S. J. Wells, "Consensus recommendations on diagnostic testing for the detection of paratuberculosis in cattle in the United States," Journal of the American Veterinary Medical Association, vol. 229, no. 12, pp. 1912-1919, 2006.

[61] A. J. DaMassa, P. S. Wakenell, and D. L. Brooks, "Mycoplasmas of goats and sheep," Journal of Veterinary Diagnostic Investigation, vol. 4, no. 1, pp. 101-113, 1992. 
[62] D. C. Ruffin, "Mycoplasma infections in small ruminants," The Veterinary Clinics of North America. Food Animal Practice, vol. 17, no. 2, pp. 315-332, 2001.

[63] E. F. Cassirer, K. M. Rudolph, P. Fowler, V. L. Coggins, D. L. Hunter, and M. W. Miller, "Evaluation of ewe vaccination as a tool for increasing bighorn lamb survival following pasteurellosis epizootics," Journal of Wildlife Diseases, vol. 37, no. 1, pp. 49-57, 2001.

[64] K. A. Brogden, D. Rose, R. C. Cutlip, H. D. Lehmkuhl, and J. G. Tully, "Isolation and identification of mycoplasmas from the nasal cavity of sheep," American Journal of Veterinary Research, vol. 49, no. 10, pp. 1669-1672, 1988.

[65] K. A. Brogden, H. D. Lehmkuhl, and R. C. Cutlip, "Pasteurella haemolytica complicated respiratory infections in sheep and goats," Veterinary Research, vol. 29, no. 3-4, pp. 233-254, 1998.

[66] H. Van Campen, K. Frolich, and M. Hofmann, "Pestivirus infections," in Infectious Diseases of Wild Mammals, E. Williams and I. K. Barker, Eds., pp. 232-244, Iowa State Press, Ames, Iowa, USA, 3rd edition, 2001.

[67] D. G. Pugh, Sheep and Goat Medicine, W.B.Saunders Company, Philadelphia, Pa, USA, 2002.

[68] E. E. Brako, R. W. Fulton, S. S. Nicholson, and G. F. Amborski, "Prevalence of bovine herpesvirus-1, bovine viral diarrhea, parainfluenza-3, goat respiratory syncytial, bovine leukemia, and bluetongue viral antibodies in sheep," American Journal of Veterinary Research, vol. 45, no. 4, pp. 813-816, 1984.

[69] D. K. Yang, I. J. Hwang, B. H. Kim et al., "Serosurveillance of viral diseases in korean native goats (Capra hircus)," Journal of Veterinary Medical Science, vol. 70, no. 9, pp. 977-979, 2008.

[70] R. K. Clark, D. A. Jessup, M. D. Kock, and R. A. Weaver, "Survey of desert bighorn sheep in California for exposure to selected infectious diseases," Journal of the American Veterinary Medical Association, vol. 187, no. 11, pp. 1175-1179, 1985.

[71] M. R. Dunbar, D. A. Jessup, J. F. Evermann, and W. J. Foreyt, "Seroprevalence of respiratory syncytial virus in free-ranging bighorn sheep," Journal of the American Veterinary Medical Association, vol. 187, no. 11, pp. 1173-1174, 1985.

[72] T. R. Spraker, J. K. Collins, W. J. Adrian, and J. H. Olterman, "Isolation and serologic evidence of a respiratory syncytial virus in bighorn sheep from Colorado," Journal of Wildlife Diseases, vol. 22, no. 3, pp. 416-418, 1986.

[73] W. P. Taylor, A. N. C. Okeke, and N. N. Shidali, "Prevalence of bovine virus diarrhoea and infectious bovine rhinotracheitis antibodies in Nigerian sheep and goats," Tropical Animal Health and Production, vol. 9, no. 3, pp. 171-175, 1977.

[74] M. R. Ackermann and K. A. Brogden, "Response of the ruminant respiratory tract to Mannheimia (Pasteurella) haemolytica," Microbes and Infection, vol. 2, no. 9, pp. 1079-1088, 2000.

[75] E. T. Thorne, N. Kingston, W. R. Jolley, and R. C. Bergstrom, Diseases of Wildlife in Wyoming, Wyoming Game and Fish Department, Cheyenne, Wyo, USA, 2nd edition, 1982.

[76] J. R. Georgi, Parasitology for Veterinarians, W.B.Saunders Company, Philadelphia, Pa, USA, 4th edition, 1985.

[77] E. P. Hoberg, A. Kocan, and L. G. Rickard, "Gastrointestinal strongyles in wild ruminants," in Parasitic Diseases of Wild Mammals, W. M. Samuel, M. J. Pybus, and A. Kocan, Eds., pp. 193-227, Iowa State University Press, Ames, Iowa , USA, 2nd edition, 2001.

[78] M. J. Pybus and H. Shave, "Muellerius capillaris (Mueller, 1889) (Nematoda: Protostrongylidae): an unusual finding in Rocky Mountain bighorn sheep (Ovis canadensis canadensis Shaw) in South Dakota," Journal of Wildlife Diseases, vol. 20, no. 4, pp. 284-288, 1984.
[79] V. O. Ezenwa, A. M. Hines, E. A. Archie, E. P. Hoberg, I. M. Asmundsson, and J. T. Hogg, "Muellerius capillaris dominates the lungworm community of bighorn sheep at the National Bison Range, Montana," Journal of Wildlife Diseases, vol. 46, no. 3, pp. 988-993, 2010.

[80] E. J. Jenkins, Ecological investigation of a new host-parasite relationship: paralephstrongylus odocoilei in thinhorn sheep (Ovis dalli), Ph.D. Thesis, University of Saskatchewan, Saskatoon, Canada, 2005.

[81] E. P. Hoberg, "Invasive processes, mosaics and the structure of helminth parasite faunas," OIE Revue Scientifique et Technique, vol. 29, no. 2, pp. 255-272, 2011.

[82] F. Brauer and P. Van Den Driessche, "Models for transmission of disease with immigration of infectives," Mathematical Biosciences, vol. 171, no. 2, pp. 143-154, 2001.

[83] D. Armstrong, R. Jakob-Hoff, and U. S. Seal, Animal Movements and Disease Risk: A Workbook, Conservation Breeding Specialist Group (SSC/IUCN), Apple Valley, Minn, USA, 2001.

[84] C. Zepeda, M. Salman, and R. Ruppanner, "International trade, animal health and veterinary epidemiology: challenges and opportunities," Preventive Veterinary Medicine, vol. 48, no. 4, pp. 261-271, 2001.

[85] L. Budd, Morag Bell, and T. Brown, "Of plagues, planes and politics: controlling the global spread of infectious diseases by air," Political Geography, vol. 28, no. 7, pp. 426-435, 2009. 

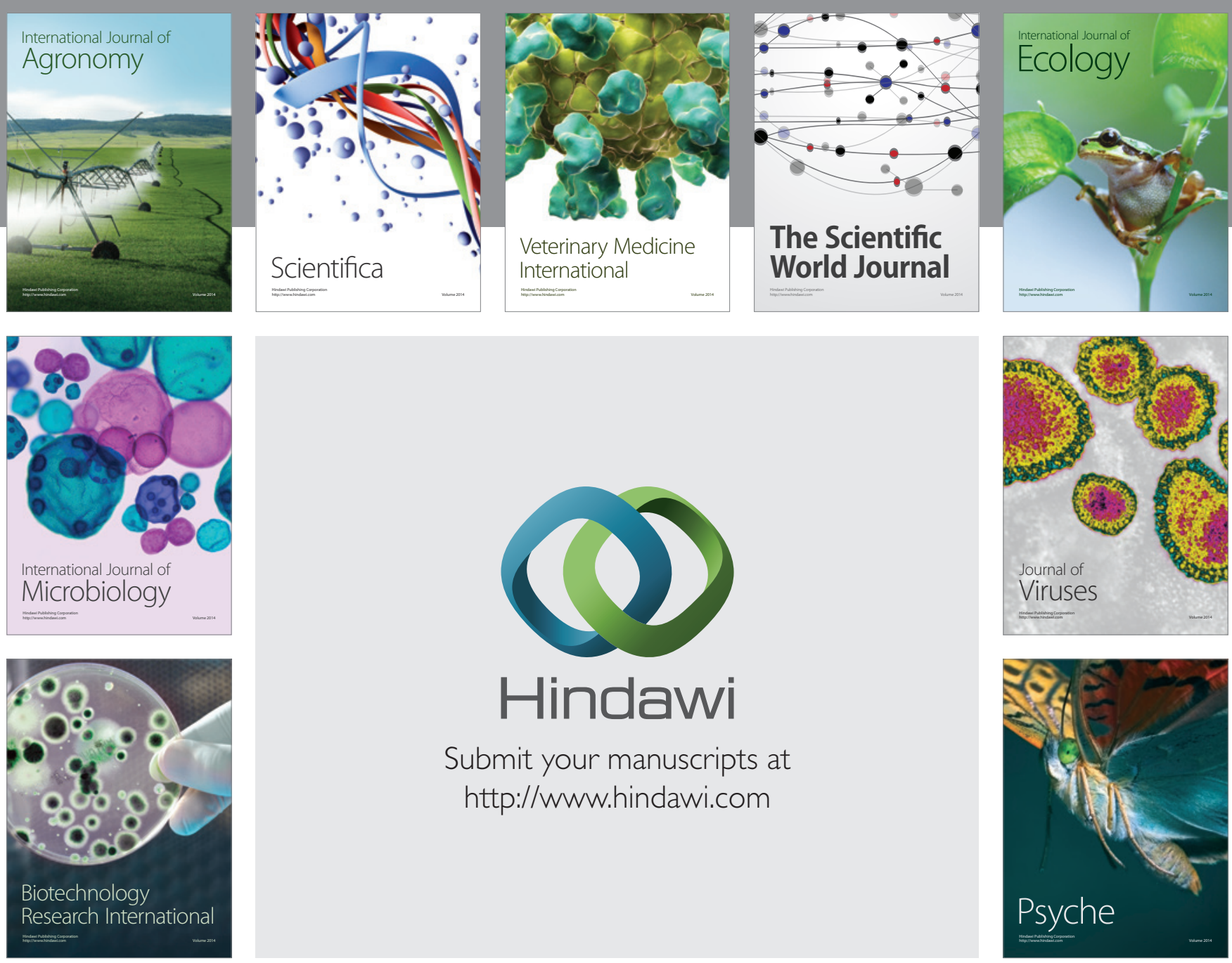

Submit your manuscripts at

http://www.hindawi.com
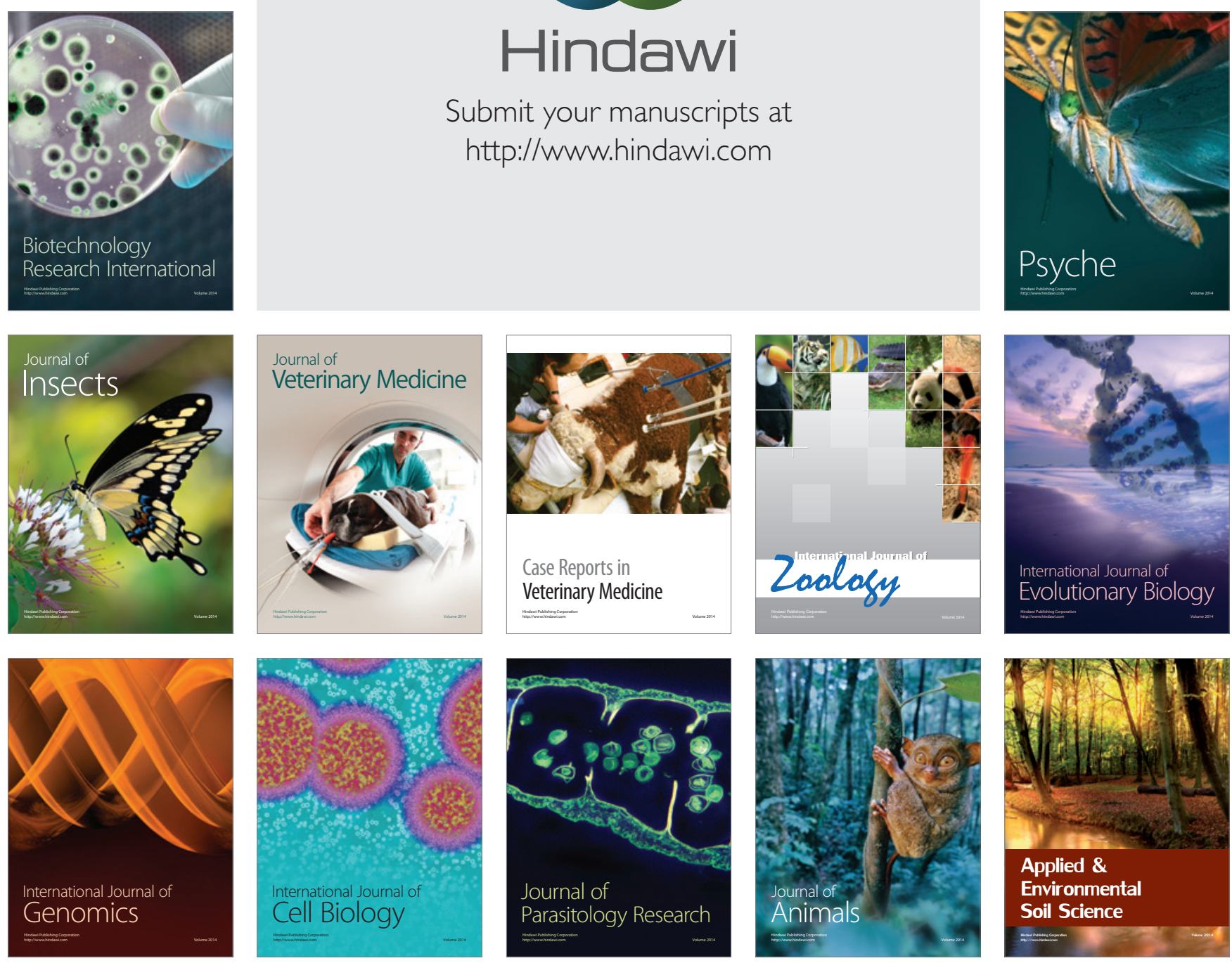